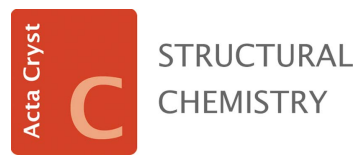

ISSN 2053-2296

Received 22 July 2015

Accepted 16 September 2015

Edited by C. S. Frampton, Brunel University, England

Keywords: space group; space-group determination; twin; metric specialization.

Supporting information: this article has supporting information at journals.iucr.org/c

\section{Methods of space-group determination - a supplement dealing with twinned crystals and metric specialization}

\author{
Howard D. Flack*
}

Chimie minérale analytique et appliquée, University of Geneva, Geneva, Switzerland. *Correspondence e-mail: crystal@flack.ch

Tables for the determination of space group for single crystals, twinned crystals and crystals with a specialized metric are presented in the form of a spreadsheet for use on a computer. There are 14 tables, one for each of the Bravais-lattice types. The content of the tables is arranged so that at the intersection of rows, displaying the conditions for reflection, and of columns, displaying the Laue and crystal classes, one finds those space groups compatible with the observed Bravais-lattice type, the conditions for reflection and the Laue and crystal classes. The tables are intended to be of direct use to an experimentalist working with an unknown structure.

\section{Introduction}

In the fifth edition of International Tables for Crystallography, Vol. A, Space-group symmetry (Hahn, 2002), hereafter ITA, there is an excellent, somewhat dated, section on the Determination of Space Group (Looijenga-Vos \& Buerger, 2002). For the sixth edition of ITA, a new chapter titled Methods of space-group determination has been written (Shmueli et al., 2015). Moreover, a separate version of this latter text, omitting the more advanced material but including four real didactic examples, has also been prepared as a submission to the Brief Teaching Edition of ITA. However, all of this material is applicable only to single-crystal samples. The current text provides a supplement to cover many cases of crystalline samples that are twinned and also those that have a specialized metric.

The current effort to extend the texts cited above owes much to the recent publication of two papers, namely Effects of merohedric twinning on the diffraction pattern (Nespolo et al., 2014) and Partial order among the 14 Bravais types of lattices: basics and applications (Grimmer, 2015). Without the work of these authors, the present publication would not have been undertaken.

This text is very definitely constructed as a supplement to Shmueli et al. (2015). As space-group determination from twins is an advanced topic, introductory material is not presented here. The reader is referred to Twinning of Crystals (Hahn \& Klapper, 2003) and to Shmueli et al. (2015) for a grounding in these matters. Relevant theory may be found in Nespolo et al. (2014), Grimmer (2015) and Shmueli et al. (2015). The basic steps in the process of space-group determination are presented here in outline with the sole purpose of accentuating the similarities and differences in the observations and treatment of single and twinned crystals. 
Table 1

Example of diffraction symmetry for various values of the twin fractions.

Crystal class $\overline{4}$. Twinning operations are $1,2_{[100]}, \overline{1}, m_{[100]}$ generating domains called $\mathrm{d} 1, \mathrm{~d} 2, \mathrm{~d} 3$ and $\mathrm{d} 4$, respectively, with twin fractions $v_{\mathrm{d} 1}, v_{\mathrm{d} 2}, v_{\mathrm{d} 3}$ and $v_{\mathrm{d} 4}$, and $v_{\mathrm{d} 1}+v_{\mathrm{d} 2}+v_{\mathrm{d} 3}+v_{\mathrm{d} 4}=1$.

\begin{tabular}{lllll}
\hline$v_{\mathrm{d} 1}$ & $v_{\mathrm{d} 2}$ & $v_{\mathrm{d} 3}$ & $v_{\mathrm{d} 4}$ & $\begin{array}{l}\text { Diffraction } \\
\text { symmetry }\end{array}$ \\
\hline arbitrary & arbitrary & arbitrary & arbitrary & $\overline{4}$ \\
0.5 & 0.5 & 0.0 & 0.0 & $\overline{4} 2 \mathrm{~m}$ \\
0.5 & 0.0 & 0.5 & 0.0 & $4 / \mathrm{m}$ \\
0.5 & 0.0 & 0.0 & 0.5 & $\overline{4} \mathrm{~m} 2$ \\
0.25 & 0.25 & 0.25 & 0.25 & $4 / \mathrm{mmm}$ \\
\hline
\end{tabular}

\section{Symmetry information from the diffraction pattern}

Following the procedure presented in \$1.6.2.1 of Shmueli et al. (2015), the first stage in the experimental procedure of spacegroup determination provides one or several observed Bravais-lattice types from the positions of the diffraction maxima in reciprocal space by the process of cell reduction. For a single crystal without metric specialization, the observed Bravais-lattice type should be that of the crystal under investigation. For a twinned crystal, this may not be the case. For example, a crystal with an $h R$ Bravais-lattice type twinned by a twofold rotation about the unique axis will display an $h P$ Bravais-lattice type. A crystal with a specialized metric is another example. A crystal with an $o C$ Bravais-lattice type with metrical relation $a=\sqrt{3} b$ will display an $h P$ Bravaislattice type. Tables 3 and 4 of Grimmer (2015) contain the relevant information. In short, the observed Bravais-lattice type may not be that of the crystal under study but nevertheless provides useful information for the process of spacegroup determination.

The second stage of the procedure is to determine the Laue class and, under favourable conditions, the crystal class of the crystal from an examination of the symmetry of the intensities of Bragg reflections in a diffraction pattern. For a single crystal, the Laue and crystal class of the diffraction data should be that of the crystal. This is not true for twinned crystals. Nespolo et al. (2014, and references therein) provide the necessary theory. The intersection group of the point groups of the several domains in a twinned crystal provides the minimum point-group symmetry of the diffraction pattern. The corresponding Laue group is the minimal centrosymmetric supergroup of this intersection group. However, further symmetry may be added to this intersection group by relations amongst the mass (or volume) fractions of the twin domains. For example, a complete twin (i.e. all possible twin domains present) with identical twin fractions will display the pointgroup symmetry of the holohedry. The point-group symmetry of the diffraction pattern of a twinned crystal depends on the values of the twin fractions. As an example, consider a crystal in crystal class $\overline{4}$. Its holohedry is $4 / \mathrm{mmm}$. Twinning operations are $1,2_{[100]}, \overline{1}, m_{[100]}$ generating domains called $\mathrm{d}_{1}, \mathrm{~d}_{2}, \mathrm{~d}_{3}$ and $\mathrm{d}_{4}$ respectively, with twin fractions $v_{\mathrm{d} 1}, v_{\mathrm{d} 2}, v_{\mathrm{d} 3}$ and $v_{\mathrm{d} 4}$, and $v_{\mathrm{d} 1}+$ $v_{\mathrm{d} 2}+v_{\mathrm{d} 3}+v_{\mathrm{d} 4}=1$. Under each of these twinning operations the point group $\overline{4}$ is invariant, so the intersection group is $\overline{4}$
Table 2

Reflection conditions for a crystal in the space group $P c a 2_{1}$ (No. 29) with a tetragonal metric $a=b, c, \alpha=\beta=\gamma=90^{\circ}$.

Domain 1 refers to a single crystal in the standard orientation. Domain $4_{[001]}$ refers to a single-crystal rotated by $90^{\circ}$ about [001] from the standard orientation. The final line of the table refers to a twinned crystal containing the two domains.

\begin{tabular}{llllll}
\hline $\begin{array}{l}\text { Reflection } \\
\text { conditions }\end{array}$ & $0 k l$ & $h 0 l$ & $h 00$ & $0 k 0$ & $00 l$ \\
\hline Domain 1 & $l=2 n$ & $h=2 n$ & $h=2 n$ & & $l=2 n$ \\
Domain 4 $_{[001]}$ & $k=2 n$ & $l=2 n$ & & $k=2 n$ & $l=2 n$ \\
Twin $1+4_{[001]}$ & $k=2 n$ or $l=2 n$ & $h=2 n$ or $l=2 n$ & & & $l=2 n$ \\
\hline
\end{tabular}

itself. Table 1 shows the diffraction symmetry displayed for various values of the twin fractions. Another example is given by the case of a crystal in crystal class $m m 2$ (Bravais-lattice type $o P$ ), but with a specialized metric $a=b$, giving a metrical symmetry of $4 / \mathrm{mmm}$. If the twofold axis of $\mathrm{mm} 2$ is parallel to the fourfold axis of $4 / \mathrm{mmm}$, then the point group $\mathrm{mm} 2$ is maintained by all of the operations of $4 / \mathrm{mmm}$ and the intersection group is $m m 2$ itself. On the other hand, if the twofold axis of $m m 2$ is perpendicular to the fourfold axis of $4 / \mathrm{mmm}$, only one $m$ plane of $m m 2$ is maintained by the operations in $4 / \mathrm{mmm}$ and the intersection group is $m$.

The third stage of the procedure uses the conditions for reflection, commonly called the systematic absences. The conditions for reflection for each domain in the twinned crystal are fixed by the space group of the structure in the orientation of each domain. As the domains are in various orientations, it results that the conditions for reflection for one domain may correspond to or annihilate those of the other domains. Consequently, the conditions for reflection for the twinned crystal may not correspond to any space group indicated in part 7 of ITA. Take as an example, a crystal structure in the space group $P c a 2_{1}$ (No. 29), for which the cell dimensions display $a=b, c, \alpha=\beta=\gamma=90^{\circ}$. Thus, the crystal structure has an $o P$ Bravais-lattice type in the orthorhombic family, but the metric indicates a $t P$ Bravais-lattice type in the tetragonal family. Table 2 displays the reflection conditions for a single crystal of this structure both in the standard orientation and in one rotated by $90^{\circ}$ about [001] from the standard orientation, and, in the final line, the reflection conditions for a twinned crystal containing the two domains. As the reflection condition on $00 l$ is identical in the two domains, this condition is maintained in the twin. A reflection condition on $h 00$ occurs for the basic domain but not for the rotated one. Consequently, the reflection condition on $h 00$ is annihilated in the twin, likewise for $0 k 0$. Reflection conditions on $0 k l$ (and $h 0 l$ ) occur for both domains but are different in form. For the twin, these conditions have to be combined by an or operation. There is no single crystal which displays the reflection conditions of this twin.

\section{Selection of twin laws}

The content of the tables given in the Supporting information is restricted in various ways which will now be described. 


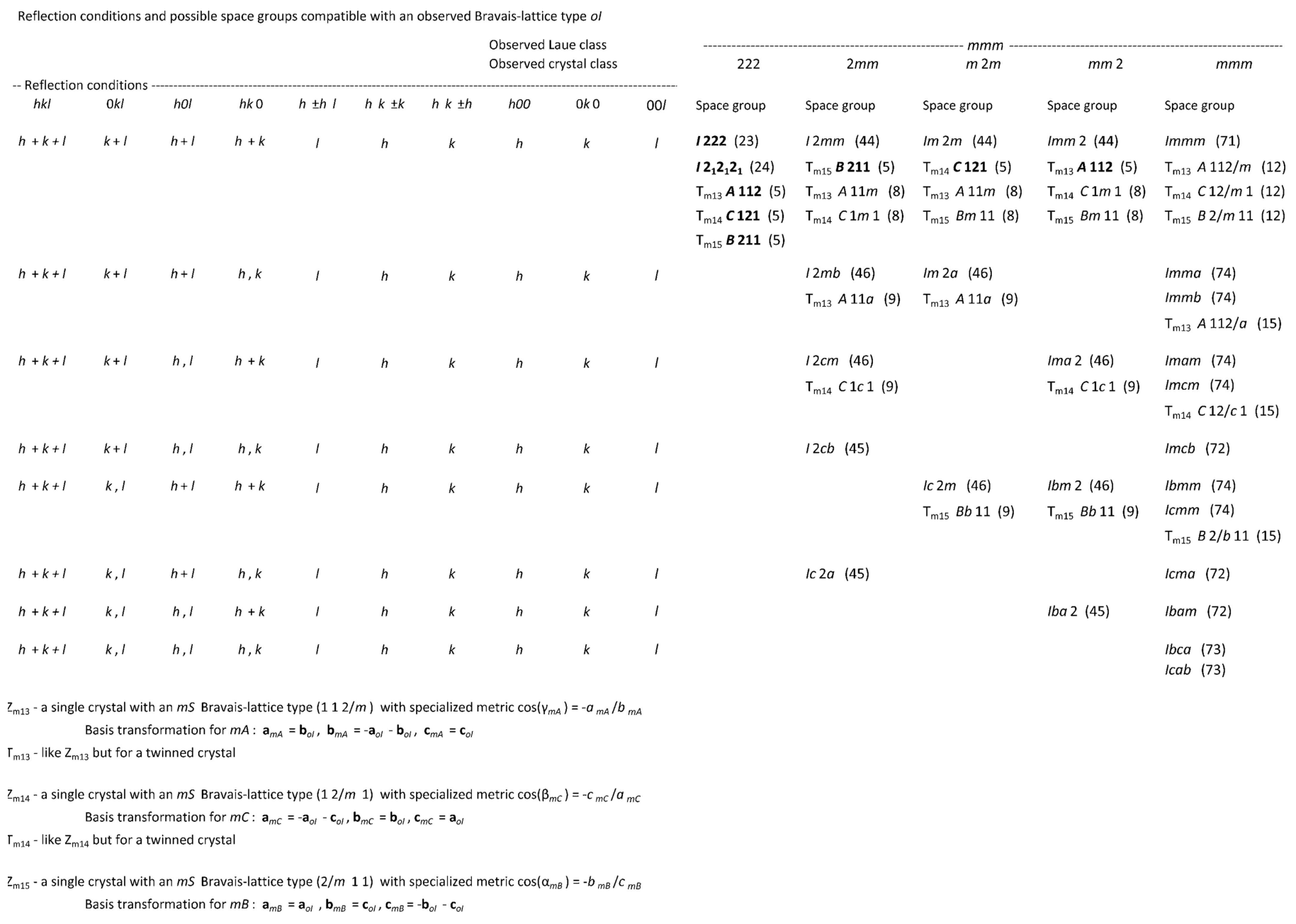

Figure 1

Top left-hand part of the Table for Bravais-lattice type $o I$.

The tables apply to diffraction patterns which can be indexed by triples of integers, $h k l$, using one single reciprocal (and consequently direct) lattice. Diffraction patterns with split reflections or reflections which separate as the temperature is changed are not dealt with directly. It may however be possible to index them on several reciprocal lattices of the same metric and subsequently to generate a single-reciprocallattice data set. Crystals with modulated structures and quasicrystals are not dealt with in this text. In short, the tables apply both to single crystals and to twinned crystals.

The classes of twins that have been included in our tables are essentially the same as those enumerated in the tables and text of Nespolo et al. (2014). However, there are omissions and additions in the current text. Included in the current tables are all cases with a twin-lattice index $[\mathrm{j}]=1$, with or without metric specialization. However, two common cases of $[\mathrm{j}]=3$ are also included. These are ones in which a twin twofold rotation is parallel to a threefold axis of the crystal class. These latter do not require any metric specialization and occur in the $h R, c P$, $c F$ and $c I$ Bravais-lattice types.

Nespolo et al. (2014) deal first with classes of twins in which there is a group-subgroup relation between the crystal point group and its holohedry. The figure of group-subgroup relationships amongst the three-dimensional point groups, given in Fig. 10.1.3.2 of Hahn \& Klapper (2002), is most useful in such an analysis. Such relations impose no additional restriction on the lattice metric other than those due to the holohedry itself, i.e. no metric specialization is required.

Nespolo et al. (2014) also deal with twinning associated with metric specialization, e.g. a crystal in the tetragonal family but displaying cell dimensions $a=b=c$ and $\alpha=\beta=\gamma=90^{\circ}$. In such cases, the lattice metric would indicate one Bravaislattice type (in the cubic family in the above example), whereas the crystal really has a lower one (in the tetragonal family in the above example). The hierarchical arrangement of Bravais-lattice types is wonderfully displayed in Fig. 2 of Grimmer (2015). The tables in our Supporting information only include cases of a metric specialization corresponding to line segments without intermediate nodes in Fig. 2 of Grimmer (2015). The tables of Nespolo et al. (2014) have a few cases where the metric specialization covers several line segments with intermediate nodes.

Twinning by inversion is given a special (non)treatment in the Supporting information. This enables the tables to be 
shortened. The justification is as follows. Twinning by inversion never affects the conditions for reflection and the Laue class. The crystal class of a crystal twinned by inversion with equal volumes for the two domains will be centrosymmetric and other instances will be noncentrosymmetric. The weakness of Friedel differences (i.e. $\left.|F(h k l)|^{2}-|F(\overline{h k l})|^{2}\right)$ and systematic errors and random uncertainties in the intensity measurements make it difficult in general to observe the true crystal class. Moreover, it is common practice to treat twinning by inversion at the least-squares refinement stage for any noncentrosymmetric crystal structure by way of the Flack (1983) parameter. Consequently, in our Supporting information, noncentrosymmetric space groups always appear in one or several of the noncentrosymmetric point groups in the corresponding Laue class.

\section{Description of the tables}

It is helpful to study the text of this section in conjunction with Fig. 1 which shows the top left hand part of the table for Bravais-lattice type $o I$.

The tables are presented in a form for direct use by the experimentalist wishing to determine the possible space group(s) of a crystal. The spreadsheet containing the tables is available in the Supporting information and at http://crystal. flack.ch/TwinMSGD.xls. The spreadsheet contains 14 tables, each table corresponding to one of the Bravais-lattice types. Each table is labelled by the symbol of its Bravais-lattice type. Each Bravais-lattice type is assumed to be presented in its conventional setting.

In each table, the columns on the left-hand side present sets of reflection conditions. The reflection indices of the subset of reflections to which each reflection condition applies is indicated in the column heading. Thus, $h k 0$ means that the first two reflection indices may take any integer value, independent one of the other, whereas the third one must be zero. In these tables, there are no implicit rules governing the reflection indices. Thus, $h h l$ means that the first two indices must be identical. Thus, $h h l$ does not apply to reflections with indices $h \bar{h} l$. A separate column, headed $h \bar{h} l$, gives the conditions on the $h \bar{h} l$ reflections. Should the reflection conditions on $h h l$ and $h \bar{h} l$ be identical, a column headed $h \pm h l$ may be used. For brevity, some of the reflection conditions have been abbreviated, as indicated in full in the index of the tables. In brief, a ',' means 'and', a '/' means 'or' and a relation with no '=' should be interpreted as ' $=2 n$ '. All space groups compatible with a set of reflection conditions are presented in the same row as the reflection conditions. However, any row many extend over several lines of the table.

Entries of space groups in the table for each crystal class are arranged in single columns. The columns of crystal classes belonging to the same Laue class are placed adjacent to one another. The symbols of the crystal and Laue classes refer to the conventional basis set of the observed Bravais-lattice type.

Entries at the intersection of a row and a column indicate those space groups which are compatible with the observed Bravais-lattice type (of the table), with the crystal and Laue class of the column, and with the reflection conditions of the row. Entries which appear as a Hermann-Mauguin symbol (without any prefixes) refer to single crystals with the indicated space group. Entries which are preceded by a Greek character refer to twinned crystals without any metric specialization. The interpretation of the Greek character is given at the foot of the table (unfortunately there are no such examples in Fig. 1). Entries which are preceded by the character ' $Z$ ' with a subscripted suffix refer to single crystals with a specialized metric. The interpretation of the ' $Z$ ' character is given at the foot of the table (unfortunately there are no such examples in Fig. 1). Finally, entries which are preceded by the character ' $\mathrm{T}$ ' with a subscripted suffix refer to twinned crystals with a specialized metric. The interpretation of the ' $\mathrm{T}$ ' character is given at the foot of the table.

All space groups on a conventional choice of unit cell are included in the tables in the Supporting information. All alternative settings displayed in Part 7 of ITA are thus included. The following further alternative settings, not displayed in Part 7 of ITA, are also included: space group $P b \overline{3}$ (No. 205) and all the space groups with an $h R$ Bravais lattice in the reverse setting on hexagonal axes. As an aid in the study of naturally-occurring macromolecules and compounds made by enantioselective synthesis, the space groups of enantiomerically pure compounds (Sohncke space groups) are typeset in bold.

\subsection{Construction of the Tables}

The tables were constructed as a result of some labour (Hirshfeld, 1968). All of the published works cited above were of considerable help and value. However, it was in the derivation of the intersection groups of twin domains and the enumeration of the reflection conditions for space groups on nonconventional bases and settings that occasioned the most work. No tested software could be identified to automate this effort. Consequently, the derivations were undertaken by hand (or perhaps more correctly, by head) using both geometric and algebraic techniques. The author accepts full responsibility for any errors or omissions in the tables.

\section{Detection of twinning}

Various ancillary techniques are helpful in the determination of space groups. These have been covered in full for single crystals by Shmueli et al. (2015) and are also applicable to twinned crystals and crystals with a specialized metric. For the determination of the space group of a twinned crystal, one also needs information on the methods available for the detection of twins. This can be used to make an informed selection between the space groups of single and twinned crystals which are displayed in the tables of the Supporting information. Fortunately, the subject has been given rather full coverage in various works in a style which is of immediate use by an experimentalist. These will be cited here but the content will not be described in detail. 
Redinbo \& Yeates (1993) have a section on the detection of twinning in a paper describing the structure determination of a macromolecule. On reading Redinbo \& Yeates (1993) (and other macromolecular papers), one has always to bear in mind that compounds such as this are known, or presumed, to be enantiomerically pure, implying that the space group is restricted to one of the 65 Sohncke space groups. Follow-ups are available in Yeates \& Fam (1999) and Padilla \& Yeates (2003). In the conclusion of their article on the refinement of twinned structures, Herbst-Irmer \& Sheldrick (1998) give a list of 12 characteristic warning signs for twinning. The context of their article is small-molecule crystallography. Chandra et al. (1999) make an interesting point concerning the analysis of the packing density. Finally, the first three sections of Dauter (2003) give very good coverage of the subject.

\section{References}

Chandra, N., Acharya, K. R. \& Moody, P. C. E. (1999). Acta Cryst. D55, 1750-1758.

Dauter, Z. (2003). Acta Cryst. D59, 2004-2016.

Flack, H. D. (1983). Acta Cryst. A39, 876-881.

Grimmer, H. (2015). Acta Cryst. A71, 143-149.
Hahn, T. (2002). Editor. International Tables for Crystallography, Vol. A, Space-group symmetry, 5th ed. Dordrecht/Boston/London: Kluwer Academic Publishers.

Hahn, T. \& Klapper, H. (2002). Point groups and crystal classes, edited by $\mathrm{T}$. Hahn, in International Tables for Crystallography, Vol. A, Space-group symmetry, 5th ed. Dordrecht/Boston/London: Kluwer Academic Publishers.

Hahn, T. \& Klapper, H. (2003). Twinning of crystals, edited by A. Authier, in International Tables for Crystallography, Vol. D, Physical Properties of Crystals, 1st ed. Dordrecht/Boston/London: Kluwer Academic Publishers.

Herbst-Irmer, R. \& Sheldrick, G. M. (1998). Acta Cryst. B54, 443-449. Hirshfeld, F. L. (1968). Acta Cryst. A24, 301-311.

Looijenga-Vos, A. \& Buerger, M. J. (2002). Determination of Space Group, edited by T. Hahn, in International Tables for Crystallography, Vol. A, Space-group symmetry, 5th ed. Dordrecht/ Boston/London: Kluwer Academic Publishers.

Nespolo, M., Ferraris, G. \& Souvignier, B. (2014). Acta Cryst. A70, 106-125.

Padilla, J. E. \& Yeates, T. O. (2003). Acta Cryst. D59, 1124-1130.

Redinbo, M. R. \& Yeates, T. O. (1993). Acta Cryst. D49, 375-380.

Shmueli, U., Flack, H. D. \& Spence, J. C. H. (2015). Methods of spacegroup determination, edited by M. Aroyo, in International Tables for Crystallography, Vol. A, Space-group symmetry, 6th ed. London: Wiley

Yeates, T. O. \& Fam, B. C. (1999). Structure, 7, R25-R29. 


\section{supporting information}

Acta Cryst. (2015). C71, 916-920］doi:10.1107/S2053229615017325]

Methods of space-group determination - a supplement dealing with twinned crystals and metric specialization

Howard D. Flack

Computing details 